\title{
NUMERICAL SOLUTION OF TEMPERATURE DEPENDENT THERMAL CONDUCTIVITY ON MHD FREE CONVECTION FLOW WITH JOULE HEATING ALONG A VERTICAL WAVY SURFACE
}

\author{
N. Parveen" and M. A. Alim \\ Department of Mathematics, Bangladesh University of Engineering and Technology, Dhaka-1000, Bangladesh \\ *Corresponding e-mail: nazma@math.buet.ac.bd
}

\begin{abstract}
Temperature dependent thermal conductivity on magnetohydrodynamic (MHD) free convective flow of viscous incompressible fluid with Joule heating along a uniformly heated vertical wavy surface has been investigated numerically. The governing nonlinear boundary layer equations are mapped into a domain of a vertical flat plate and solved by an implicit finite difference method known as Keller-box scheme. The skin friction coefficient, the rate of heat transfer in terms of local Nusselt number, the stream lines and the isotherms are reported for different parameter combinations.
\end{abstract}

Keywords: Joule heating, MHD, temperature dependent thermal conductivity, free convection, uniform surface temperature, Keller-box method, wavy surface

\section{INTRODUCTION}

Joule heating effect of the laminar free convection flow of an electrically conducting fluid and heat transfer problem has been presented by many investigators because of its considerable practical applications. It is also necessary to study the heat transfer from an irregular surface because irregular surfaces are often present in many applications, such as radiator, heat exchangers and heat transfer enhancement devices. The thermal conductivity of the fluid to be proportional to a linear function of temperature, two semi-empirical formulae which was proposed by Charraudeau ${ }^{1} \mathrm{Yao}^{2,3}$ first investigated the natural convection heat transfer from an isothermal vertical wavy surface and used an extended Prantdl's transposition theorem and a finite-difference scheme. He proposed a simple transformation to study the natural convection heat transfer for an isothermal vertical sinusoidal surface. These simple coordinate transformations method to change the wavy surface into a flat plate. Moulic and $\mathrm{Yao}^{4}$ also investigated also investigated natural convection along wavy surface with uniform heat flux. Alam et al. ${ }^{5}$ have also studied the problem of free convection from a wavy vertical surface in presence of a transverse magnetic field. On the other hand, the combined effects of thermal and mass diffusion on the natural convection flow of a viscous incompressible fluid along a vertical wavy surface have been investigated by Hossain and Rees ${ }^{6}$. In this paper the effect of waviness of the surface on the heat and mass flux has been investigated in combination with the species concentration for a fluid having Prandtl number equal to 0.7 . Hossain and $\mathrm{Munir}^{7}$ investigated the natural convection flow of a viscous fluid about a truncated cone with temperature dependent viscosity and thermal conductivity. Natural convection with variable viscosity and thermal conductivity along a vertical wavy cone have been investigated by Munir et al. ${ }^{8}$. Hossain et al. ${ }^{9}$ considered natural convection of fluid with temperature dependent viscosity from heated vertical wavy surface. Wang and Chen ${ }^{10}$ investigated transient force and free convection along a vertical wavy surface in micropolar fluid. Natural and mixed convection heat and mass transfer along a vertical wavy surface have been investigated by Jang et al. ${ }^{11,12}$. Molla et al. ${ }^{13}$ have studied natural convection flow along a vertical wavy surface with uniform surface temperature in presence of heat generation/absorption. Tashtoush and $\mathrm{Al}$-Odat ${ }^{14}$ investigated magnetic field effect on heat and fluid flow over a wavy surface with a variable heat flux. $\mathrm{Yao}^{15}$ also studied natural convection along a vertical complex wavy surface. Rahman et al. ${ }^{16}$ investigated the effects of temperature dependent thermal conductivity on MHD free convection flow along a vertical flat plate with heat conduction. Rahman and Alim $^{17}$ considered numerical study of magnetohydrodynamic free convective heat transfer flow along a vertical plate with temperature dependent thermal conductivity. Recently, Parveen and Alim ${ }^{18}$ investigated Joule heating effect on MHD natural convection flow along a vertical wavy surface with viscosity dependent on temperature. At the same time Parveen and $\mathrm{Alim}^{19}$ considered effect of temperature dependent thermal conductivity on magnetohydrodynamic natural convection flow along a vertical wavy surface. It is known that thermal conductivity may be change significantly with temperature. For a liquid, it has been found that the thermal conductivity $k$ varies with temperature in an approximately linear manner in the range from 0 to $400^{\circ} \mathrm{F}\left(\right.$ see $\mathrm{Kays}^{20}$ ).

The above literatures survey it is found that Joule heating effect on MHD free convection flow of an 
electrically conducting fluid variation of thermal conductivity with temperature along a vertical wavy surface has not been well investigated. Main objective of the present study is detailed numerically investigation of the temperature dependent thermal conductivity on MHD free convection flow with Joule heating along a vertical wavy surface. The governing partial differential equations are reduced to locally non-similar partial differential forms by adopting some appropriate transformations. The transformed boundary layer equations are solved numerically using implicit finite difference scheme together with Keller box technique ${ }^{21}$. The surface shear stress in terms of local skin friction coefficient and the rate of heat transfer in terms of local Nusselt number, the stream lines as well as the isotherms are shown graphically for different values of thermal conductivity parameter $\gamma$, Joule heating $J$, magnetic parameter $M$ and the amplitude-to-length ratio of the wavy surface $\alpha$ while Prandtl number $\operatorname{Pr}=0.73$ (air).

\section{MATHEMATICAL FORMULATION}

Consider a steady two dimensional natural convection flow of an electrically conducting viscous and incompressible fluid with variable thermal conductivity along a vertical wavy surface. Over the work it is assumed that the surface temperature of the vertical wavy surface $T_{w}$ is uniform, where $T_{w}>T_{\infty}$. The boundary layer analysis outlined below allows $\bar{\sigma}(X)$ being arbitrary, but our detailed numerical work assumed that the surface exhibits sinusoidal deformations. The wavy surface may be described by

$Y_{w}=\bar{\sigma}(X)=\alpha \sin \left(\frac{n \pi X}{L}\right)$

where $L$ is the wave length associated with the wavy surface.

Under the usual Boussinesq approximation, the continuity, momentum and energy equations can be written as:

$$
\begin{aligned}
& \frac{\partial U}{\partial X}+\frac{\partial V}{\partial Y}=0 \\
& U \frac{\partial U}{\partial X}+V \frac{\partial U}{\partial Y}=-\frac{1}{\rho} \frac{\partial P}{\partial X} \nabla^{2} U+g \beta\left(T-T_{\infty}\right)+\frac{\delta_{0} \beta_{0}^{2}}{\rho} U \\
& U \frac{\partial V}{\partial X}+V \frac{\partial V}{\partial Y}=-\frac{1}{\rho} \frac{\partial P}{\partial Y}+v \nabla^{2} V \\
& U \frac{\partial T}{\partial X}+V \frac{\partial T}{\partial Y}=\frac{1}{\rho C_{p}} \nabla \cdot(k \nabla T)+\frac{\delta_{0} \beta_{0}^{2}}{\rho C_{p}} U^{2}
\end{aligned}
$$

where $(X, Y)$ are the dimensional coordinates along and normal to the tangent of the surface and $(U, V)$ are the velocity components parallel to $(X, Y)$, $\nabla^{2}\left(=\partial^{2} / \partial x^{2}+\partial^{2} / \partial y^{2}\right) \quad$ is the Laplacian operator, $g$ is the acceleration due to gravity, $P$ is the dimensional pressure of the fluid, $\rho$ is the density, $\beta_{0}$ is the strength of magnetic field, $\delta_{0}$ is the electrical conduction, $k(T)$ is the thermal conductivity of the fluid in the boundary layer region depending on the fluid temperature, $\beta$ is the coefficient of thermal expansion, $v(=\mu / \rho)$ is the kinematics viscosity, $\mu$ is the dynamic viscosity and $C_{p}$ is the specific heat due to constant pressure.

The boundary conditions relevant to the above problem are

$U=0, V=0, T=T_{w} \quad$ at $Y=Y_{w}=\bar{\sigma}(X)$

$U=0, \quad T=T_{\infty}, \quad P=p_{\infty} \quad$ as $\quad Y \rightarrow \infty$

where $T_{w}$ is the surface temperature, $T_{\infty}$ is the ambient temperature of the fluid and $p_{\infty}$ is the pressure of fluid outside the boundary layer.

The geometry of the wavy surface and the twodimensional Cartesian coordinate system are shown in Fig. 1.

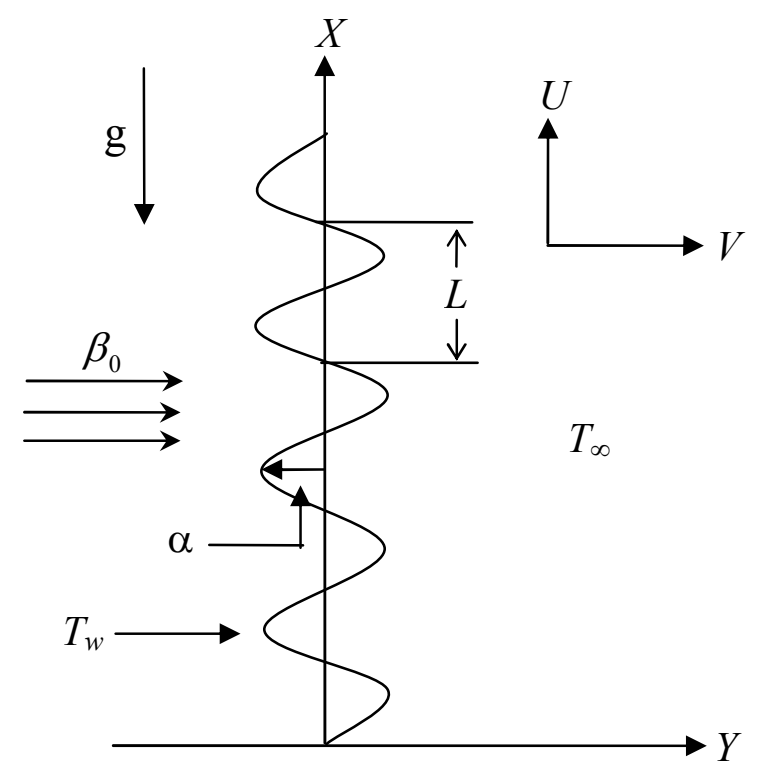

Figure 1. The coordinate system and the physical model

The variable thermal conductivity chosen in this study that is introduced by Charraudeau ${ }^{1}$ and used by Hossain and Munir $^{7}$ as follows:

$k=k_{\infty}\left[1+\gamma^{*}\left(T-T_{\infty}\right)\right]$

where $k_{\infty}$ is the thermal conductivity of the ambient fluid and $\gamma^{*}=\frac{1}{k_{f}}\left(\frac{\partial k}{\partial T}\right)_{f}$ is a constant.

Using Prandtl's transposition theorem to transform the irregular wavy surface into a flat surface as extended by $\mathrm{Yao}^{3}$ and boundary-layer approximation, the following dimensionless variables are introduced for non-dimensionalizing the governing equations, 


$$
\begin{aligned}
& x=\frac{X}{L}, \quad y=\frac{Y-\bar{\sigma}}{L} G r^{\frac{1}{4}}, p=\frac{L^{2}}{\rho v^{2}} G r^{-1} P \\
& u=\frac{\rho L}{\mu} G r^{-1 / 2} U, \quad v=\frac{\rho L}{\mu} G r^{-1 / 4}\left(V-\sigma_{x} U\right) \\
& \sigma_{x}=\frac{d \bar{\sigma}}{d \bar{x}}=\frac{d \sigma}{d x}, G r=\frac{g \beta\left(T_{w}-T_{\infty}\right)}{v^{2}} L^{3}, \quad \theta=\frac{T-T_{\infty}}{T_{w}-T_{\infty}}
\end{aligned}
$$

where $\theta$ is the non-dimensional temperature function and $(u, v)$ are the dimensionless velocity components parallel to $(x, y)$. Introducing the above dimensionless dependent and independent variables into equations (2)-(5), the following dimensionless form of the governing equations are obtained after ignoring terms of smaller orders of magnitude in $G r$, the Grashof number defined in (8).

$\frac{\partial u}{\partial x}+\frac{\partial v}{\partial y}=0$

$u \frac{\partial u}{\partial x}+v \frac{\partial u}{\partial y}=-\frac{\partial p}{\partial x}+G r^{1 / 4} \sigma_{x} \frac{\partial p}{\partial y}+\left(1+\sigma_{x}^{2}\right) \frac{\partial^{2} u}{\partial y^{2}}$

$-M u+\theta$

$\sigma_{x}\left(u \frac{\partial u}{\partial x}+v \frac{\partial u}{\partial y}\right)=-G r^{1 / 4} \frac{\partial p}{\partial y}+\sigma_{x}\left(1+\sigma_{x}^{2}\right) \frac{\partial^{2} u}{\partial y^{2}}$

$-\sigma_{x x} u^{2}$

$u \frac{\partial \theta}{\partial x}+v \frac{\partial \theta}{\partial y}=\frac{1}{\operatorname{Pr}}\left(1+\sigma_{x}^{2}\right)(1+\gamma \theta) \frac{\partial^{2} \theta}{\partial y^{2}}$

$+\frac{1}{\operatorname{Pr}}\left(1+\sigma_{x}^{2}\right) y\left(\frac{\partial \theta}{\partial y}\right)^{2}+J u^{2}$

where $\operatorname{Pr}=\frac{C_{p} \mu}{k_{\infty}}$ is the Prandtl number, $M=\frac{\delta_{0} \beta_{0}^{2} L^{2}}{\mu G r^{1 / 2}}$ is the magnetic parameter, $J=\frac{\delta_{0} \beta_{0}^{2} \nu G r^{1 / 2}}{\rho C_{p}\left(T_{w}-T_{\infty}\right)}$ is the Joule heating parameter and $\gamma=\gamma^{*}\left(T_{w}-T_{\infty}\right)$ is the thermal conductivity variation parameter.

It can easily be seen that the convection induced by the wavy surface is described by Eqs. (9)-(12). We further notice that, Eq. (11) indicates that the pressure gradient along the $y$-direction is $O\left(G r^{-1 / 4}\right)$, which implies that lowest order pressure gradient along $x$-direction can be determined from the inviscid flow solution. For the present problem this pressure gradient $(\partial p / \partial x=0)$ is zero. Eq. (11) further shows that $G r^{1 / 4} \partial p / \partial y$ is $O(1)$ and is determined by the left-hand side of this equation. Thus, the elimination of $\partial p / \partial y$ from Eqs. (10) and (11) leads to

$$
\begin{aligned}
& u \frac{\partial \theta}{\partial x}+v \frac{\partial \theta}{\partial y}=\frac{1}{\operatorname{Pr}}\left(1+\sigma_{x}^{2}\right)(1+\gamma \theta) \frac{\partial^{2} \theta}{\partial y^{2}} \\
& +\frac{1}{\operatorname{Pr}}\left(1+\sigma_{x}^{2}\right) \gamma\left(\frac{\partial \theta}{\partial y}\right)^{2}+J u^{2}
\end{aligned}
$$

The corresponding boundary conditions for the present problem then turn into

$$
\left.\begin{array}{l}
u=v=0, \quad \theta=1 \quad \text { at } \quad y=0 \\
u=\theta=0, \quad p=0 \quad \text { as } \quad y \rightarrow \infty
\end{array}\right\}
$$

Now we introduce the following transformations to reduce the governing equations to a convenient form:

$$
\psi=x^{3 / 4} f(x, \eta), \quad \eta=y x^{-1 / 4}, \quad \theta=\theta(x, \eta)
$$

where $f(\eta)$ is the dimensionless stream function, $\eta$ is the pseudo similarity variable and $\psi$ is the stream function that satisfies the continuity equation and is related to the velocity components in the usual way as

$u=\frac{\partial \psi}{\partial y}, \quad v=-\frac{\partial \psi}{\partial x}$

Introducing the transformations given in Eq. (15) into Eqs. (13) and (12) are transformed into the new co-ordinate system. Thus the resulting equations are

$$
\begin{aligned}
& \left(1+\sigma_{x}^{2}\right) f^{\prime \prime \prime}+\frac{3}{4} f f^{\prime \prime}-\left(\frac{1}{2}+\frac{x \sigma_{x} \sigma_{x x}}{1+\sigma_{x}^{2}}\right) f^{\prime 2}+\frac{1}{1+\sigma_{x}^{2}} \theta \\
& -\frac{M x^{1 / 2}}{1+\sigma_{x}^{2}} f^{\prime}=x\left(f^{\prime} \frac{\partial f^{\prime}}{\partial x}-f^{\prime \prime} \frac{\partial f}{\partial x}\right) \\
& \frac{1}{\operatorname{Pr}}\left(1+\sigma_{x}^{2}\right)(1+\gamma \theta) \theta^{\prime \prime}+\frac{1}{\operatorname{Pr}}\left(1+\sigma_{x}^{2}\right) \gamma \theta^{\prime 2} \\
& +\frac{3}{4} f \theta^{\prime}+J x^{3 / 2} f^{\prime 2}=x\left(f^{\prime} \frac{\partial \theta}{\partial x}-\theta^{\prime} \frac{\partial f}{\partial x}\right)
\end{aligned}
$$

The boundary conditions as mentioned in Eq. (14) then take the form given below:

$$
\left.\begin{array}{l}
f(x, o)=f^{\prime}(x, o)=0, \quad \theta(x, o)=1 \\
f^{\prime}(x, \infty)=0, \quad \theta(x, \infty)=0
\end{array}\right\}
$$

The quantities of physical interested, namely, the shearing stress $\tau_{\mathrm{w}}$ in terms of the skin friction coefficient $C_{f x}$ and the rate of heat transfer in terms of Nusselt number $N u_{x}$ are prescribed by:

$$
\begin{aligned}
& C_{f x}=\frac{2 \tau_{w}}{\rho U^{2}} \text { and } N u_{x}=\frac{q_{w} x}{k_{\infty}\left(T_{w}-T_{\infty}\right)} \\
& \text { where } \tau_{w}=(\mu \bar{n} . \nabla \bar{u})_{y=0} \text { and } q_{w}=-k(\bar{n} . \nabla T)_{y=0}
\end{aligned}
$$

Using the transformations (15) and Eq. (21) into Eq. (20), the local skin friction coefficient $C_{f x}$ and the rate of heat transfer in terms of the local Nusselt number $N u_{x}$ takes the following form:

$$
\begin{aligned}
& C_{f x}(G r / x)^{1 / 4} / 2=\sqrt{1+\sigma_{x}^{2}} f^{\prime \prime}(x, o) \\
& N u_{x}(G r / x)^{-1 / 4}=-(1+\gamma) \sqrt{1+\sigma_{x}^{2}} \theta^{\prime}(x, o)
\end{aligned}
$$




\section{METHOD OF SOLUTION}

The transformed boundary layer equations are solved numerically with the help of implicit finite difference method together with the Keller-Box scheme $^{21}$. To begin with the partial differential equations (17) and (18) are first converted into a system of first order differential equations. Then these equations are expressed in finite difference forms by approximating the functions and their derivatives in terms of the center difference. Denoting the mesh points in the $x$ and $\eta$-plane by $x_{i}$ and $\eta_{j}$ where $i=1,2, \ldots, M$ and $j=1,2, \ldots, N$, central difference approximations are made, such that those equations involving $x$ explicitly are centered at $\left(x_{i-1 / 2}\right.$ ,$\left.\eta_{j-1 / 2}\right)$ and the remainder at $\left(x_{i}, \eta_{j-1 / 2}\right)$, where $\eta_{j-1 / 2}=$ $1 / 2\left(\eta_{j}+\eta_{j-1}\right)$ etc. The above central difference approximations reduces the system of first order differential equations to a set of non-linear difference equations for the unknown at $x_{i}$ in terms of their values at $x_{i-1}$. The resulting set of non-linear difference equations are solved by using the Newton's quasi-linearization method. The Jacobian matrix has a block-tridiagonal structure and the difference equations are solved using a block-matrix version of the Thomas algorithmthe; the details of the computational procedure have been discussed further by in the book by Cebeci and Bradshaw ${ }^{22}$. In the program test, a finer axial step size is tried and finds to give acceptable accuracy. A uniform grid of 201 points is used in $x$ - direction with $\Delta x=0.05$, while a non-uniform grid of 76 points lying between $\eta=0.0$ and 10.02 is chosen. Grid points are concentrated towards the heated surface in order to improve resolution and the accuracy of the computed values of the surface shear stress and rate of heat transfer. During the program test, the convergent criteria for the relative errors between two iterations are less $10^{-5}$.

\section{CODE VALIDATION}

A comparison of the present numerical results of the skin friction coefficient, $f^{\prime \prime}(x, 0)$ and the heat transfer, $-\theta^{\prime}(x, 0)$ with the results obtained by Hossain et al. ${ }^{9}$ is depicted in Table 1. Here, the magnetic parameter $M$, thermal conductivity parameter $\gamma$ and Joule heating parameter $J$ are ignored with different values of Prandtl number $\mathrm{Pr}=$ $(1.0,10.0$ and 25.0) is chosen. From Table 1, it is clearly seen that the present results are excellent agreement with the solution of Hossain et al. ${ }^{9}$.

\section{RESULTS AND DISCUSSION}

The present work is to analyze the Joule heating effect on MHD free convection flow of viscous incompressible fluid with temperature dependent thermal conductivity along a uniformly heated vertical wavy surface. Numerical values of local shearing stress and the rate of heat transfer are calculated from equations (22) and (23) in terms of the skin friction coefficients $C_{f x}$ and Nusselt number $N u_{x}$ respectively for a wide range of the axial distance $x$. For different values of the aforementioned parameters $\gamma, M, \alpha$ and $J$, the skin-friction coefficient $C_{f x}$, the rate of heat transfer in terms of Nusselt number $N u_{x}$, the streamlines as well as the isotherms are shown graphically in Figs. 2-8.

The belongings of thermal conductivity parameter $\gamma$ the local skin friction coefficient $C_{f x}$ and the rate of heat transfer in terms of the local Nusselt number $N u_{x}$ against $x$ from the wavy surface while $\alpha=0.3, M=$ $0.5, J=0.02$ and $\operatorname{Pr}=0.73$ are illustrated in Fig. 2(a) and 2(b) respectively. From Fig. 2, it is observed that the skin friction coefficient and the heat transfer rate increases significantly along the upward direction of the surface. Here it is concluded that for high thermal conductivity fluid the skin friction and the corresponding rate of heat transfer is large. The highest values of local skin friction coefficient $C_{f x}$ are $0.86677,0.98013,1.04351$ and 1.08529 for $\gamma=0.0$, $2.0,5.0$ and 10.0 respectively which occurs at $x=0.5$ shown in Fig. 2(a). The maximum values of rate of heat transfer are recorded to be $0.31743,0.53690$, 0.77737 for $\gamma=0.0,2.0$ and 5.0 respectively which occurs at same point of $x=0.5$ and 1.10617 for $\gamma=$ 10.0 which attain at the surface. Thus the skin friction coefficient and the rate of heat transfer increase by approximately $20 \%$ and $72 \%$ respectively when $\gamma$ changes from 0.0 to 10.0 .

The analysis of the effects of Joule heating parameter $J=(0.0,0.02,0.05$ and 0.10$)$ on the surface shear stress in terms of the local skin friction coefficient $C_{f x}$ and the rate of heat transfer in terms of the local Nusselt number $N u_{x}$ against $x$ are exposed within the boundary layer with $\alpha=0.3, M=0.02, \gamma=$ 5.0 and $\mathrm{Pr}=0.73$ in Fig. 3 . From this figure it can be seen that an increase in the Joule heating parameter $J$, the skin friction coefficient increases slowly along the upward direction of the surface. But the heat transfer rate decreases quickly along the downward direction of the surface. Because increasing values of Joule heating parameter $J$ decrease the temperature gradient at the surface. The maximum values of local skin friction coefficient $C_{f x}$ are 1.12519 and 1.12611 for $J=0.0$ and 0.10 respectively which occurs at the same value of $x=0.50$. Again the peak values of the rate of heat transfer $N u_{x}$ are 0.82074 and 0.81022 for $J=0.0$ and 0.10 respectively which occurs at different position of $x$. Finally it is seen that the local skin friction coefficient increases by approximately $0.10 \%$ and the rate of heat transfer devalues by approximately $1.30 \%$ as $J$ increases from 0.0 to 0.10 .

The variation of the local skin friction coefficient $C_{f x}$ and local rate of heat transfer $N u_{x}$ for different values of the amplitude-to-length ratio of the wavy surface $\alpha=(0.0,0.1,0.2,0.3)$ is illustrated in Fig. 4 for $\operatorname{Pr}=0.73, \gamma=5.0, J=0.02$ and $M=0.5$. From Fig. 4 it can be observed that the amplitude-to-length ratio of the wavy surface $\alpha$ leads to decrease the 
local skin friction coefficient $C_{f x}$ and local rate of heat transfer $N u_{x}$ at different position of $x$. The skin friction coefficient and the rate of heat transfer coefficient decrease by approximately $12 \%$ and $11 \%$ respectively as $\alpha$ increases from 0.0 to 0.3 . Because of increasing the surface waviness the velocity force decreases at the local points.
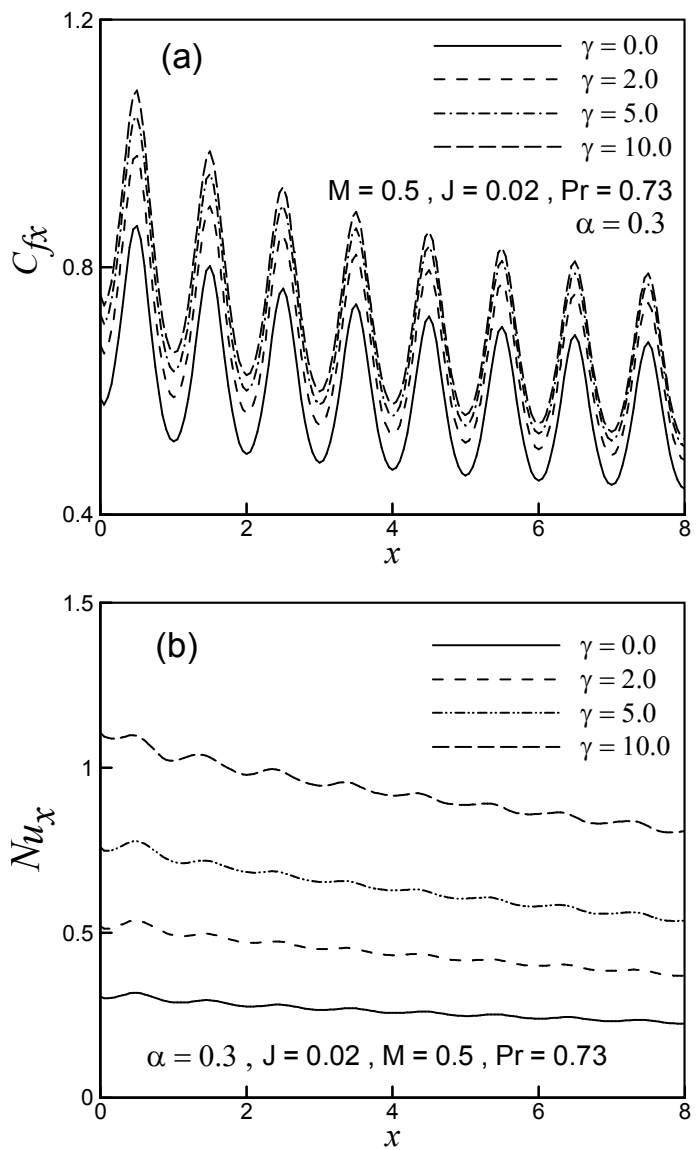

Figure 2. Variation of (a) skin friction coefficient $C_{f x}$ and (b) rate of heat transfer $N u_{x}$ against $x$ for different values of $\gamma$ with $\alpha=0.3$, $M=0.5, J=0.02$ and $\operatorname{Pr}=0.73$.

Figures 5 and 6 illustrate the effect of thermal conductivity parameter $\gamma$ on the development of streamlines and isotherms profile which are plotted for the amplitude-to-length ratio of the wavy surface $\alpha=0.3$, Prandtl number $\operatorname{Pr}=0.73, J=0.02$ and $M=$ 0.5 . The maximum values of $\psi$, that is, $\psi_{\max }$ are $6.59,11.37$ and 12.36 for thermal conductivity parameter $\gamma=0.0,5.0$ and 10.0 respectively. It is observed from Fig. 5 that as the values of $\gamma$ increases the velocity boundary layer thickness becomes higher gradually and the same result observed for the thermal boundary layer from Fig. 6. Finally it is concluded that for the effect of thermal conductivity parameter $\gamma$ the velocity of the flow and temperature of the fluid within the boundary layer increase.
The effect of Joule heating parameter $J$ equal to $0.0,0.05$ and 0.10 the streamlines and isotherms profile are depicted by the Figs. 7 and 8 respectively while Prandtl number $\operatorname{Pr}=0.73$, amplitude-to-length ratio of the wavy surface $\alpha=0.3$, thermal conductivity parameter $\gamma=5.0$ and magnetic parameter $M=0.02$. Fig. 7 depicts that the maximum values of $\psi$ increases steadily while the value of $J$ increases. The maximum values of $\psi$, that is, $\psi_{\max }$ is 13.21 for $J=0.0, \psi_{\max }$ is 14.16 for $J=0.05$ and $\psi_{\max }$ is 14.69 for $J=0.10$. From Fig. 8, it is noted that the temperature of the fluid increases for increasing values of $J$. Joule heating is the heating effect of conductors carrying currents. So velocity of the fluid flow and the temperature of the fluid increase within the boundary layer with the increasing values of $J$.
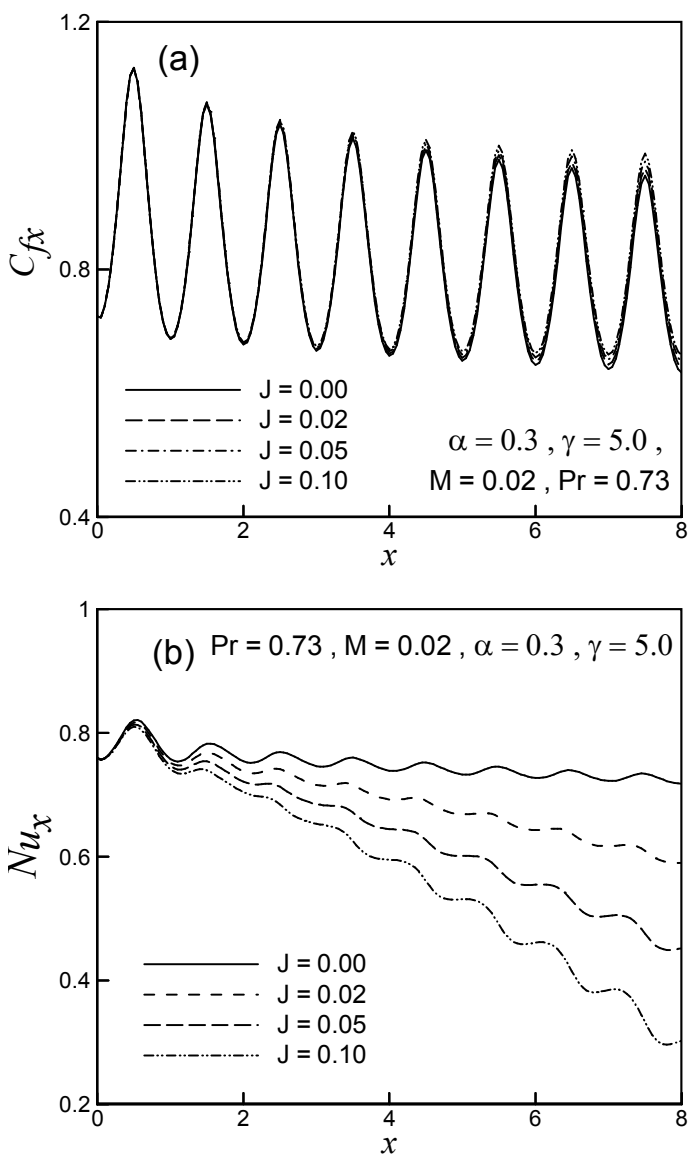

Figure 3. Variation of (a) skin friction coefficient $C_{f x}$ and (b) rate of heat transfer $N u_{x}$ against $x$ for different values of $J$ with $\alpha=0.3$, $\gamma=5.0, M=0.02$ and $\operatorname{Pr}=0.73$. 

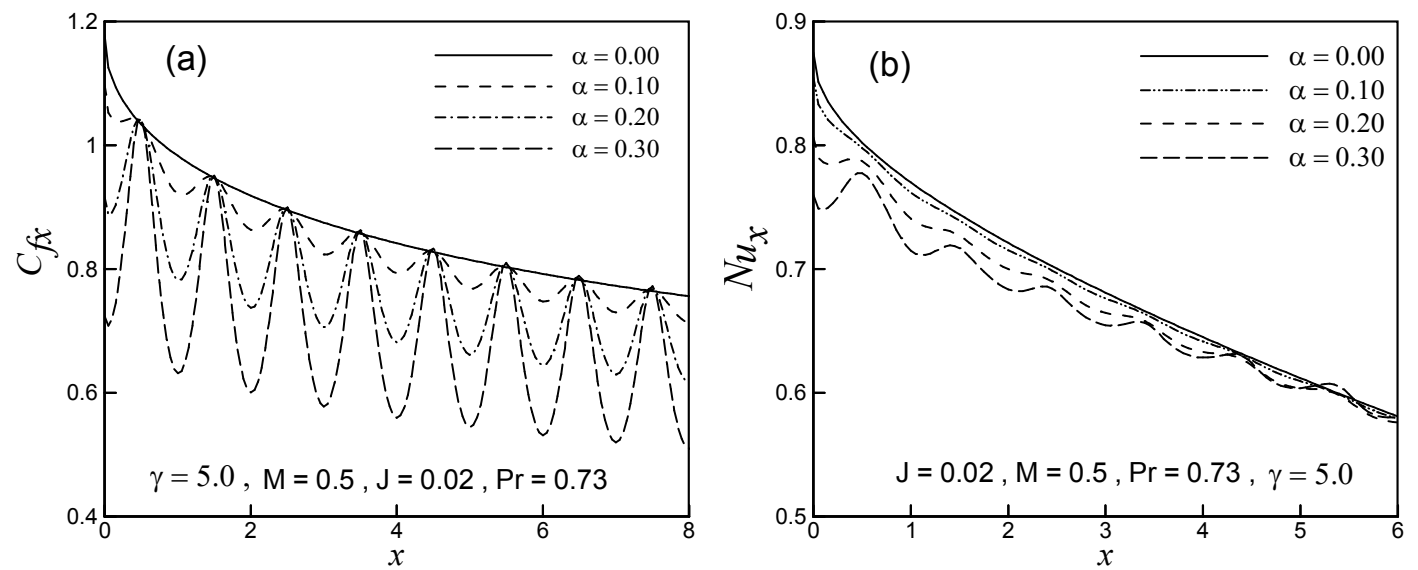

Figure 4. Variation of (a) skin friction coefficient $C_{f x}$ and (b) rate of heat transfer $N u_{x}$ against $x$ for different values of $\alpha$ with $\gamma=5.0, M=0.5, J=0.02$ and $\operatorname{Pr}=0.73$.
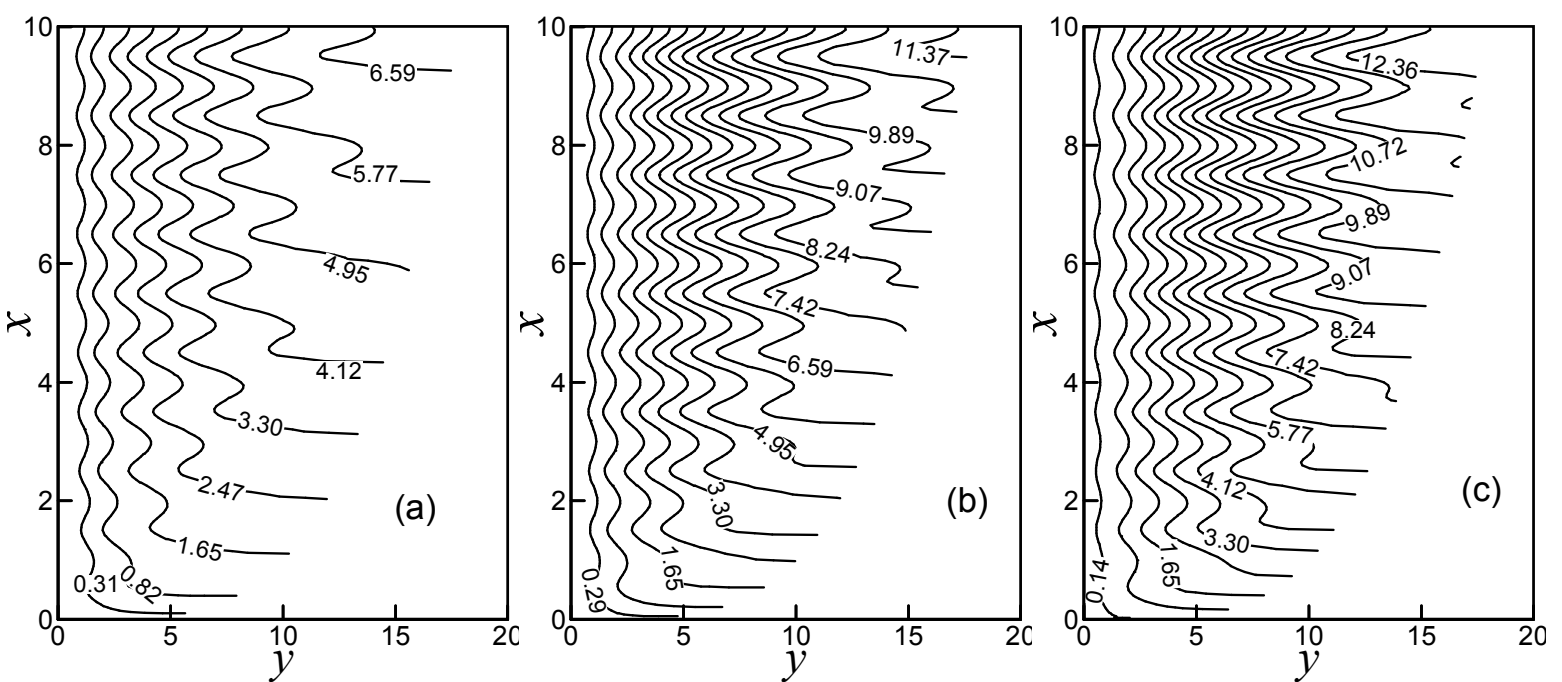

Figure 5. Streamlines for (a) $\gamma=0.0$ (b) $\gamma=5.0$ and (c) $\gamma=10.0$ while $\alpha=03, M=0.5, J=0.02$ and $\operatorname{Pr}=0.73$.
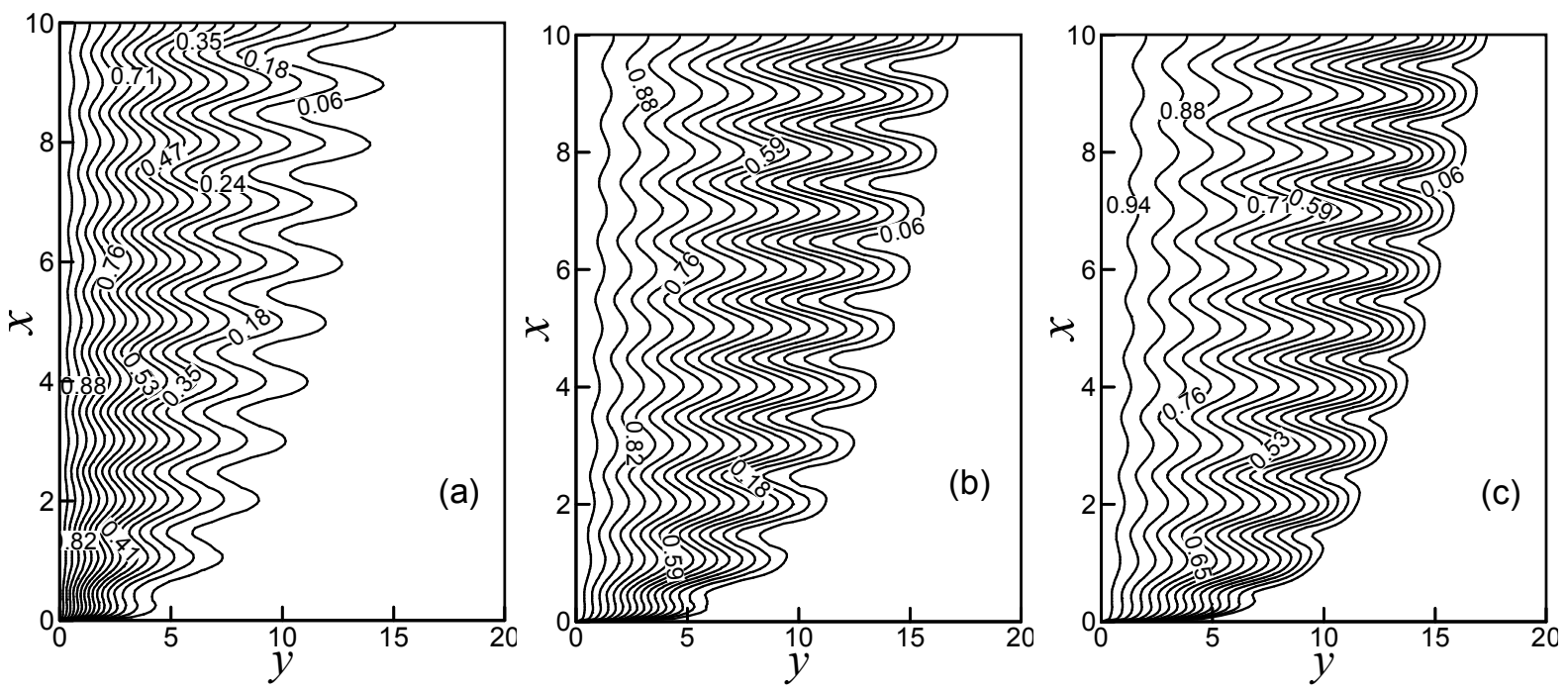

Figure 6. Isotherms for (a) $\gamma=0.0$ (b) $\gamma=5.0$ and (c) $\gamma=10.0$ while $\alpha=0.3, M=0.5, J=0.02$ and $\operatorname{Pr}=0.73$. 

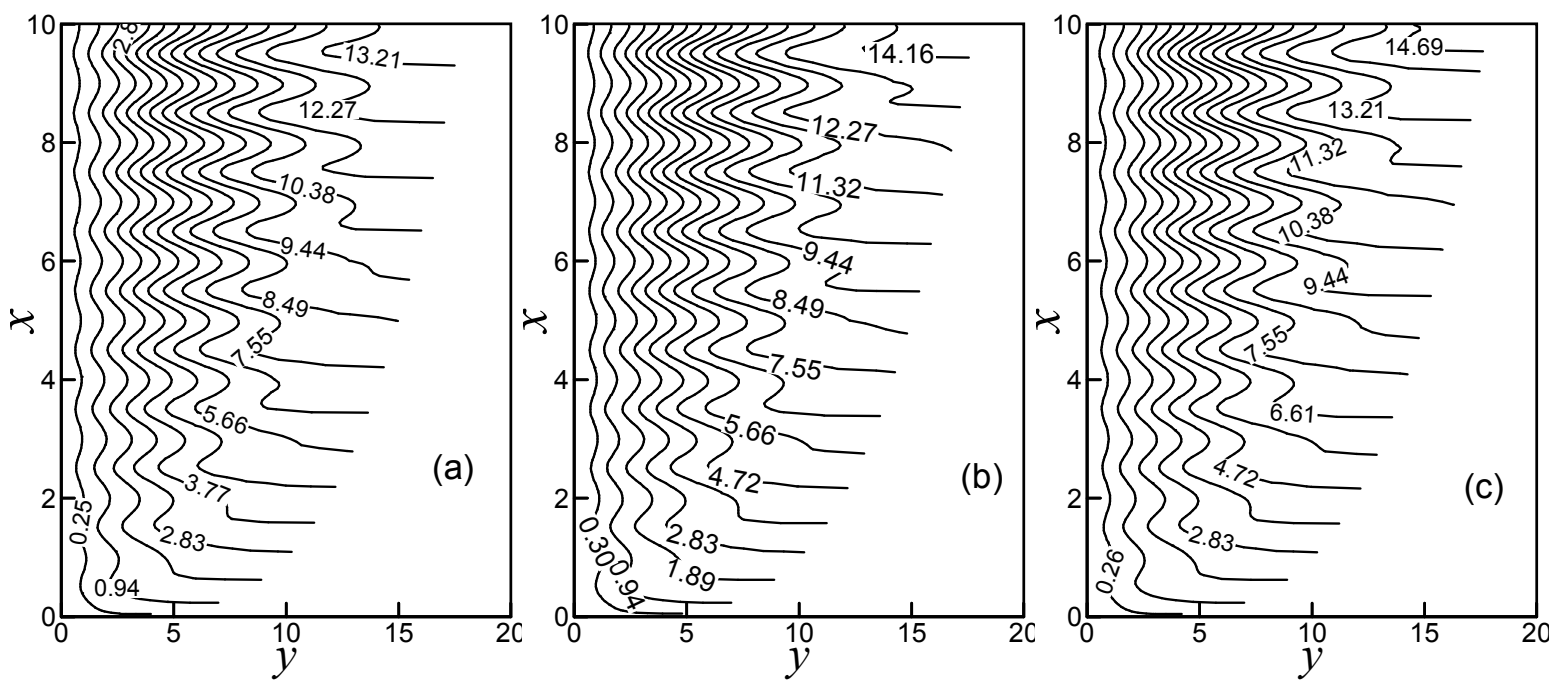

Figure 7. Streamlines for (a) $J=0.0$ (b) $J=0.05$ and (c) $J=0.10$ while $\operatorname{Pr}=0.73, M=0.02, \gamma=5.0$ and $\alpha=0.3$.
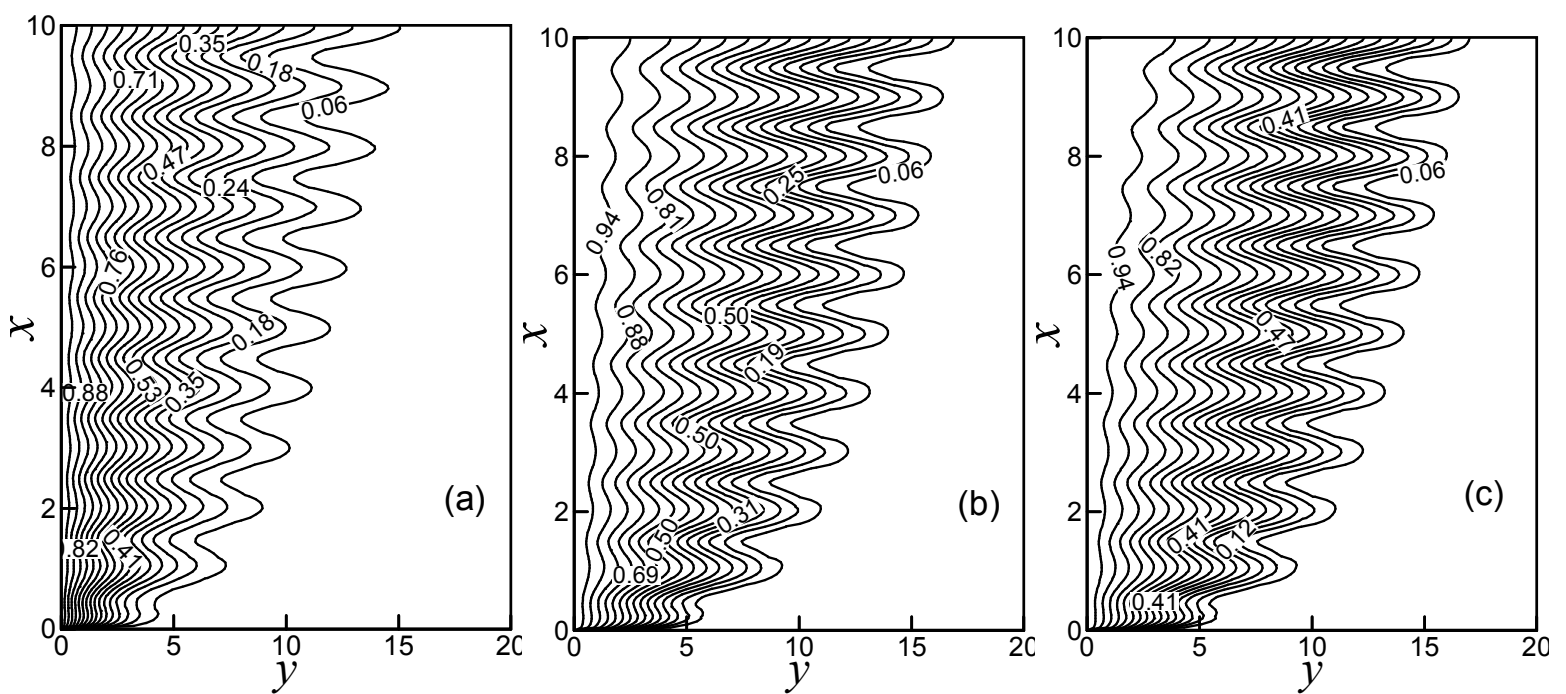

Figure 8. Isotherms for (a) $J=0.0$ (b) $J=0.05$ and (c) $J=0.10$ while $\operatorname{Pr}=0.73, M=0.02, \gamma=5.0$ and $\alpha=0.3$.

Table 1. Comparison of the present numerical results for

\begin{tabular}{|c|c|c|c|c|}
\multicolumn{4}{c}{$M=0, \gamma=0, J=0}$. \\
\hline \multirow{3}{*}{$\operatorname{Pr}$} & \multicolumn{2}{|c|}{$f^{\prime \prime}(x, 0)$} & \multicolumn{2}{c|}{$-\theta^{\prime}(x, 0)$} \\
\cline { 2 - 5 } & $\begin{array}{c}\text { Hossain et } \\
\text { al. }{ }^{9}\end{array}$ & $\begin{array}{c}\text { Present } \\
\text { work }\end{array}$ & $\begin{array}{c}\text { Hossain et } \\
\text { al. }\end{array}$ & $\begin{array}{c}\text { Present } \\
\text { work }\end{array}$ \\
\hline 1.0 & 0.908 & 0.91084 & 0.401 & 0.39914 \\
\hline 10.0 & 0.591 & 0.59482 & 0.825 & 0.82315 \\
\hline 25.0 & 0.485 & 0.48910 & 1.066 & 1.06405 \\
\hline
\end{tabular}

\section{CONCLUSION}

Temperature dependent thermal conductivity on MHD free convection flow with Joule heating along a vertical wavy surface has been studied numerically in detail. Brief summaries of the major results are listed in the following:

- For increasing values of thermal conductivity parameter $\gamma$ skin friction coefficient $C_{f x}$, local rate of heat transfer $N u_{x}$, velocity of the fluid flow and the temperature of the fluid significantly increase.
- The skin friction coefficient, velocity of the fluid flow increase and local rate of heat transfer $N u_{x}$ decreases over the whole boundary layer for increasing values of Joule heating parameter $J$. But the thermal boundary layer grows thick.

- The skin friction coefficient $C_{f x}$ and the local rate of heat transfer $N u_{x}$ decrease for increasing values of the amplitude-to-length ratio of the wavy surface.

\section{REFERENCES}

1. J. Charraudeau, Influence De Gradients De Properties Physiques En Convection Force Application $\mathrm{Au}$ Cas Du Tube, Int. J. Heat Mass Transfer, Vol. 18, pp. 87-95, 1975.

2. L. S. Yao, Natural Convection along a Vertical Wavy Surface, ASME J. Heat Transfer, Vol. 105, pp. 465-468, 1983. 
3. L. S. Yao, A note on Prandtl's Transposition Theorem, ASME J. Heat Transfer, Vol. 110, pp. 503-507, 1988.

4. S. G. Moulic and L. S. Yao, Natural Convection along Wavy Surface with Uniform Heat Flux, ASME J. Heat Transfer, Vol. 111 pp. 1106-1108, 1989.

5. K. C. A. Alam, M. A. Hossain and D. A. S. Rees, Magnetohydrodynamic Free Convection along a Vertical Wavy Surface, Int. J. Appl. Mech. Engrg, Vol. 1, pp. 555-566, 1997.

6. M. A. Hossain and D. A. S. Rees, Combined Heat and Mass Transfer in Natural Convection Flow from a Vertical Wavy Surface, Acta Mechanica, Vol. 136, pp. 133-141, 1999.

7. M. A. Hossain and M. S. Munir, Natural Convection Flow of a Viscous Fluid about a Truncated Cone with Temperature Dependent Viscosity and Thermal Conductivity, Int. J. Num. Met. Heat Fluid Flow, Vol. 11, pp. 494-510, 2001.

8. M. S. Munir, M. A. Hossain and I. Pop, Natural Convection with Variable Viscosity and Thermal Conductivity from a Vertical Wavy Cone, Int. J. Therm. Sci., Vol. 40, pp. 437-443, 2001.

9. Hossain M. A., Kabir S. and Rees D. A. S., Natural Convection of Fluid with Temperature Dependent Viscosity from Heated Vertical Wavy Surface, Z. Angew. Math. Phys., Vol. 53, pp. 48-57, 2002.

10. C. C. Wang and C. K. Chen, Transient Force and Free Convection along a Vertical Wavy Surface in Micropolar Fluid, Int. J. Heat Mass Transfer, Vol. 44, pp. 3241-3251, 2001.

11. J. H. Jang, W. M. Yan and H. C. Liu, Natural Convection Heat and Mass Transfer along a Vertical Wavy Surface, Int. J. Heat Mass Transfer, Vol. 46, pp. 1075-1083, 2003.

12. J. H. Jang and W. M. Yan, Mixed Convection Heat and Mass Transfer along a Vertical Wavy Surface, Int. J. Heat Mass Transfer, Vol. 47, pp. 419428, 2004.

13. M. M. Molla, M. A. Hossain and L. S. Yao, Natural Convection Flow along a Vertical Wavy Surface with Uniform Surface Temperature in Presence of Heat Generation/Absorption, Int. J. Therm. Sci., Vol. 43, pp. 157-163, 2004.
14. B. Tashtoush and M. Al-Odat, Magnetic Field Effect on Heat and Fluid flow over a Wavy Surface with a Variable Heat Flux, J. Magn. Magn. Mater, Vol. 268, pp. 357-363, 2004.

15. L. S. Yao, Natural Convection along a Vertical Complex Wavy Surface, Int. J. Heat Mass Transfer, Vol. 49, pp. 281-286, 2006.

16. M. M. Rahman, A. A. Mamun, M. A. Azim and M. A. Alim, Effects of Temperature Dependent Thermal Conductivity on Magnetohydrodynamic Free Convection Flow along a Vertical Flat Plate with Heat Conduction, Nonlinear Analysis: Modell. and Cont., Vol. 13 (4), pp. 513-524, 2008.

17. M. M. Rahman and M. A. Alim, Numerical Study of Magnetohydrodynamic Free Convective Heat Transfer flow along a Vertical Plate with Temperature Dependent Thermal Conductivity, J. of Naval Architecture and Marine Engineering, Vol. 6, No. 1, pp. 16-29, 2009.

18. N. Parveen and M. A. Alim, Joule Heating Effect on Magnetohydrodynamic Natural Convection flow along a Vertical Wavy Surface with Viscosity Dependent on Temperature, Int. J. Energy Technology, Vol. 3, pp. 1-10, 2011.

19. N. Parveen and M. A. Alim, Effect of Temperature Dependent Thermal Conductivity on Magnetohydrodynamic Natural Convection flow along a Vertical Wavy Surface, Int. J. Energy Technology, Vol. 3 (9), pp. 1-9, 2011.

20. W. M. Kays, Convective Heat and Mass Transfer, McGraw-Hill, New York, 1966, p. 362.

21. H. B. Keller, Numerical Methods in Boundary Layer Theory, Ann. Rev. Fluid Mech., Vol. 10, pp. 417-433, 1978.

22. T. Cebeci and P. Bradshaw, Physical and Computational Aspects of Convective Heat Transfer, Springer, New York, 1984. 